\title{
Mejoramiento de un programa informático para la selección de nuevas tecnologías de control de arena de tipo mecánico en campos operados por Ecopetrol S.A.
}

\author{
Gustavo Adolfo Beltrán Ladino, Juan Sebastián Carvajal Hernández \\ Universidad de América \\ Código Postal: 110111. Bogotá - Colombia
}

E-mail: gustavo07beltran@outlook.com,juanchiscar2@hotmail.com

\begin{abstract}
Resumen
En los campos petrolíferos de Colombia ocurre que, debido al arenamiento, se presenta corrosión, erosión y taponamiento de equipos y tuberías de superficie y de subsuelo, incrementando así la frecuencia de trabajos de intervención a los pozos productores de hidrocarburos. Dado lo anterior, surgió la necesidad de mejorar el módulo de control de arena, compuesto por dos herramientas del Manual General de Completamiento de Pozos ya existente (MGCP) para recomendar cuatro nuevas tecnologías de control de arena para su uso en yacimientos que presenten areniscas altamente no uniformes y rangos de tamaño de grano fino. Las mejoras introducidas a dicho módulo permitieron que dentro de la primera herramienta (matriz teórica para los campos de estudio) se recomendaran estas cuatro nuevas tecnologías para su uso en 22 de los 24 campos estudiados por autores previos, pertenecientes a cinco cuencas de Colombia. Así mismo, la segunda herramienta también fue actualizada mediante la introducción de las cuatro nuevas tecnologías mecánicas, además de incluirse un segundo parámetro de selección (tamaño de grano promedio) para recomendar el uso de tecnologías mecánicas nuevas o convencionales de control de arena con base en el último parámetro ya mencionado y en el ya existente coeficiente de uniformidad.
\end{abstract}

Palabras clave: Completamiento, Tecnologías de tipo mecánico, Control de arena, Manual General de Completamiento de Pozos.

\section{Improvement of a computer program for the selection of new mechanical-type sand control technologies in fields operated by Ecopetrol S.A.}

\begin{abstract}
In Colombian oil fields it occurs that, due to the sanding, there is a corrosion, erosion and plugging of the equipment and the surface and subsoil pipes, thus increasing the frequency of intervention work in the hydrocarbon producing wells. Given the above, the need to improve the sand control module arose. This one is composed by two tools from the already existing General Manual of Wells Completion (MGCP), in order to recommend four new sand control technologies for their usage in reservoirs with both highly non-uniform sandstones and fine grain size ranges. The improvements introduced to this module allowed that within the first tool (theoretical matrix for the studied oilfields) these four new technologies were recommended for their usage in 22 of the 24 fields studied by previous authors, belonging to five Colombian basins. In addition, the second tool was updated by introducing these four new mechanic technologies and including a second selection parameter (average grain size) for recommending the use of an either new or conventional mechanic tool of sand control based on both the previously mentioned parameter and the already existing coefficient of uniformity.
\end{abstract}

Keywords: Completion, Mechanical type technologies, Sand control, General Manual of Wells Completion.

Cita: Beltrán Ladino, G. A. y Carvajal Hernández, J. S. (2019). Mejoramiento de un programa informático para la selección de Nuevas Tecnologías de Control de Arena de Tipo Mecánico en campos operados por Ecopetrol S.A. Revista Fuentes: El reventón energético, 17(1), 55-69. 


\section{Introducción}

La producción de arena es un problema serio que se presenta cuando las fuerzas desestabilizadoras superan la resistencia mecánica de la roca, ya sea por condiciones naturales u operacionales e interviene en muchos de los activos de petróleo y gas a nivel mundial (Guardia et al, 2011), esto debido a que afecta drásticamente los regímenes de producción de hidrocarburos (Tamayo et al, 2017) alterando la integridad de los equipos en fondo, submarinos e instalaciones en superficie con problemas tales como corrosión, taponamiento y acumulación de arena en los equipos y dificultad de disponer estos granos en superficie, que en su conjunto implicará un costo de miles de millones de dólares por año (Páez et al, 2016).

Una solución a esta problemática es la instalación de los dispositivos de control de arena de tipo mecánico. Este proyecto tiene el objetivo de generar un programa informático mejorado para la selección de nuevas tecnologías de control de arena de tipo mecánico mediante la incorporación de cuatro nuevas tecnologías a dos herramientas web. El Frac Pack, una técnica que permite el fracturamiento y control de arena de forma simultánea reduciendo el daño cercano al pozo (Quintero et al, 2010; Ballesteros, 2016); la malla expandible, adaptable a geometrías irregulares de pozo mediante su expansión; la malla sinterizada, altamente resistente a la abrasión y corrosión y por último la malla MeshRite $^{\mathrm{TM}}$ que retiene partículas mayores a 30 micras y adicionalmente presenta $40 \%$ de área disponible para el flujo de hidrocarburos.

Estas tecnologías en su conjunto permitirán mejorar la toma de decisiones para el completamiento de pozos en Ecopetrol S.A. generando un posible aumento en la tasa de producción de hidrocarburos al permitir un factible incremento en la eficiencia de las operaciones de control de arena debido a la ampliación del rango de selección de las nuevas tecnologías y la aplicabilidad de un método adecuado de control para pozos nuevos y completados.

\section{Metodología}

Inicialmente se abordan los parámetros utilizados para recomendación de mecanismos nuevos o convencionales de control de arena de tipo mecánico ya definidos previamente por González y Ramírez (2016). Acto seguido, se describe de forma conceptual el Manual General de Completamiento de Pozos (MGCP), el módulo de control de área y las dos herramientas modificadas. En segunda instancia son definidas las tecnologías a incorporar y sus parámetros de operación. Finalmente, el procedimiento efectuado para la modificación de las dos herramientas.

1. Distribución granulométrica del yacimiento. Esta puede determinarse mediante análisis por tamizado o de partícula con láser (LPS) (Bellarby, 2009). En la primera técnica, una muestra de formación previamente limpiada y disgregada es pasada a través de una serie de tamices sacudidos continuamente para diferenciar tamaños comprendidos entre 2350 y 44 micras. Luego, los granos de tamaño arena retenidos son pesados para determinar sus porcentajes de peso según su tamaño y elaborar una gráfica representativa de distribución granulométrica que confronte los tamaños de grano con sus respectivos porcentajes (Penberthy \& Shaughnessy, 1992).

Mediante el análisis de partícula con láser, la muestra es introducida a un analizador LPS que mide el tamaño de grano por medio de un detector que utiliza la dispersión de la luz. Posteriormente, se emplean percentiles para obtener una descripción precisa de la clasificación de los tamaños de grano de la formación analizada (Bellarby, 2009).

Coeficiente de uniformidad. Es el cociente presentado entre los percentiles d40 y d90 de una prueba granulométrica (ver Ecuación 1 y Tabla 1) (Xiang, 2003).

$$
U=\frac{d 40}{d 90}
$$

A continuación, se indican las variables de la anterior ecuación:

$\mathrm{U}=$ Coeficiente de uniformidad.

$\mathrm{d} 40=$ Percentil 40 de la distribución granulométrica del yacimiento.

d90= Percentil 90 de la distribución granulométrica del yacimiento.

Tabla 1. Característica de la arenisca productora según el coeficiente de uniformidad. (Penberthy y Shaughnessy,

1992).

\begin{tabular}{|c|c|}
\hline Coeficiente de uniformidad & Característica \\
\hline $\mathrm{U}<3$ & Uniforme \\
\hline $3<\mathrm{U}<5$ & No uniforme \\
\hline $\mathrm{U}>5$ & Altamente no uniforme \\
\hline
\end{tabular}


Escala de Wentworth. Es una escala granulométrica utilizada para clasificar los sedimentos (ver Tabla 2), siendo las partículas de tamaño mayor a $64 \mathrm{~mm}$ clasificadas como cantos rodados y las menores de 0,0039 $\mathrm{mm}$ correspondientes a arcilla (Schlumberger, 2018).

Tabla 2. Clasificación de los granos de arena de acuerdo con su tamaño (Jackson \& Richardson, 2017).

\begin{tabular}{|c|c|}
\hline Sedimento & Diámetro [Micras] \\
\hline Arena muy gruesa & $1001-2000$ \\
\hline Arena gruesa & $501-1000$ \\
\hline Arena media & $251-500$ \\
\hline Arena fina & $126-250$ \\
\hline Arena muy fina & $62-125$ \\
\hline
\end{tabular}

Rango de tamaño de ranura (RTR). Aplica para la recomendación del tamaño de ranuras del liner ranurado y/o mallas. El cálculo del tamaño se basó en cuatro autores, Coberly, Coberly actualizado, Penberthy y Shaughnessy y Regent Energy (González \& Ramirez, 2016).

$$
\begin{gathered}
\text { RTR Coberly }=(2 * d 10) / 25.400 \\
\text { RTR Coberly act. }=\frac{2 * d 10}{25.400} \\
\text { RTR Penberthy }=\frac{2 * d 10}{25.400} \\
\text { RTR Regent Energy }=\frac{2 * d 10}{25.400}
\end{gathered}
$$

A continuación, se indican las variables de las ecuaciones anteriores:

d10 $=$ Percentil 10 de la distribución granulométrica del yacimiento.

Definición del tamaño de grava. conforme con Penberthy y Shaughnessy (1992), el criterio de Saucier (1974) es el más utilizado por los ingenieros. En este se determina que el tamaño de la grava debe poseer un tamaño comprendido entre 5 y 6 veces del tamaño medio del grano de la formación en su percentil 50 .

$$
D 50=5 \sim 6 * d 50
$$

A continuación, se indican las variables de la anterior ecuación:

D50= Tamaño medio de grava.

d50 = Tamaño medio del grano de la formación en su percentil 50 .
Para efectos del presente trabajo, será tenido en cuenta este último criterio, el cual fue empleado por González y Ramírez (2016) para calcular un tamaño de grava seis veces mayor al tamaño de grano de la formación en su percentil 50 .

Manual General de Completamiento de Pozos (MGCP). Es una plataforma informática mediante la cual el usuario obtiene una recomendación para la posible instalación de herramientas en fondo de pozo de acuerdo con el trabajo de completamiento que se planea ejecutar, luego de ingresar al aplicativo unos datos específicos relacionados con dicha intervención. El programa informático es ejecutado mediante lenguaje PHP. Para efectos de este artículo, solo se abordará el módulo de control de arena, ya que en este fueron implementadas las 4 tecnologías.

Módulo de control de arena. Este se compone de dos herramientas. En primer lugar, posee una matriz de selección preliminar de mecanismos de control de arena, en la cual se recomienda una tecnología nueva o contemporánea a veinticuatro campos de Colombia a partir del coeficiente de uniformidad y el rango de tamaño de grano de las formaciones productoras presentes en cada campo; los valores de estos parámetros fueron recopilados por González y Ramírez a partir de mediciones realizadas a muestras de yacimiento (González \& Ramírez, 2016). En segundo lugar, cuenta con una herramienta que permite la recomendación de un mecanismo de control de arena nuevo o contemporáneo para un pozo nuevo en función a datos granulométricos de entrada.

Matriz de selección preliminar de mecanismos de control de arena en los campos operados por Ecopetrol S.A. Esta matriz permite la identificación de mecanismos de control de arena instalados actualmente en 24 campos pertenecientes a cinco cuencas de Colombia (Llanos Orientales, Valle Medio del Magdalena, Valle Superior del Magdalena, Catatumbo y Putumayo) y la recomendación de tecnologías convencionales o nuevas de tipo mecánico a partir de variables granulométricas. Para su desarrollo, se tuvo en cuenta la matriz existente (González \& Ramírez, 2016), donde se utilizó el coeficiente de uniformidad y el rango de tamaño de grano para la selección y recomendación de tecnologías de control de arena de tipo mecánico. Además, de recomendar una nueva tecnología, la matriz contiene unas recomendaciones independientes para cada uno de los 24 campos justificando la 
implementación de la tecnología recomendada. Las tecnologías mecánicas convencionales de control de arena ya existentes en la matriz son el empaquetamiento con grava, el liner ranurado, la malla convencional y la malla premium.

Herramienta de selección de mecanismos de control de arena. Esta herramienta permite el ingreso de datos de tamaños de grano tanto de la formación productora como de la arena producida al Manual General de Completamiento de pozos (MGCP) para obtener, posteriormente, la recomendación de una tecnología mecánica de control de arena con base en el coeficiente de uniformidad y el tamaño de grano promedio, los cuales son calculadas por el aplicativo web. Así mismo, este modelo también determina tamaños de ranura para las mallas recomendadas y el tamaño de grava en caso de requerirse un empaquetamiento con grava.

2. Definición y parámetros operacionales de las nuevas tecnologías de control de arena de tipo mecánico. A continuación, se presentan los conceptos y parámetros de operación de las nuevas tecnologías con base en diferentes casos de éxito a nivel mundial y a fichas técnica de fabricantes. Es importante aclarar que se hará especial énfasis en el coeficiente de uniformidad y rango de tamaño de grano debido a que únicamente se tiene información de estos dos parámetros para las cuencas del estudio. Adicionalmente, se presentan los demás parámetros de forma complementaria, para que sirvan como criterios de referencia para recomendar una tecnología que satisfaga sus problemas de arenamiento.

Frac Pack. Esta técnica involucra el fracturamiento hidráulico (ver Figura 1 y Tabla 3) de un yacimiento mediante el bombeo de un fluido de alta viscosidad (presión superior a la de fractura) y el empaquetamiento de grava de las fracturas rellenando el anular entre el cemento y la formación (Norman, 2004).

Malla Expandible (ESS). Esta malla (ver Figura 2 y Tabla 4) consta de una estructura de tubo de base ranurada, expandible y adaptable a las paredes del hoyo, un medio filtrante compuesto de alambre metálico tejido, y una capa externa de protección y encapsulación (Salehi-Moorkani \& Ghasemzadeh, 2011).

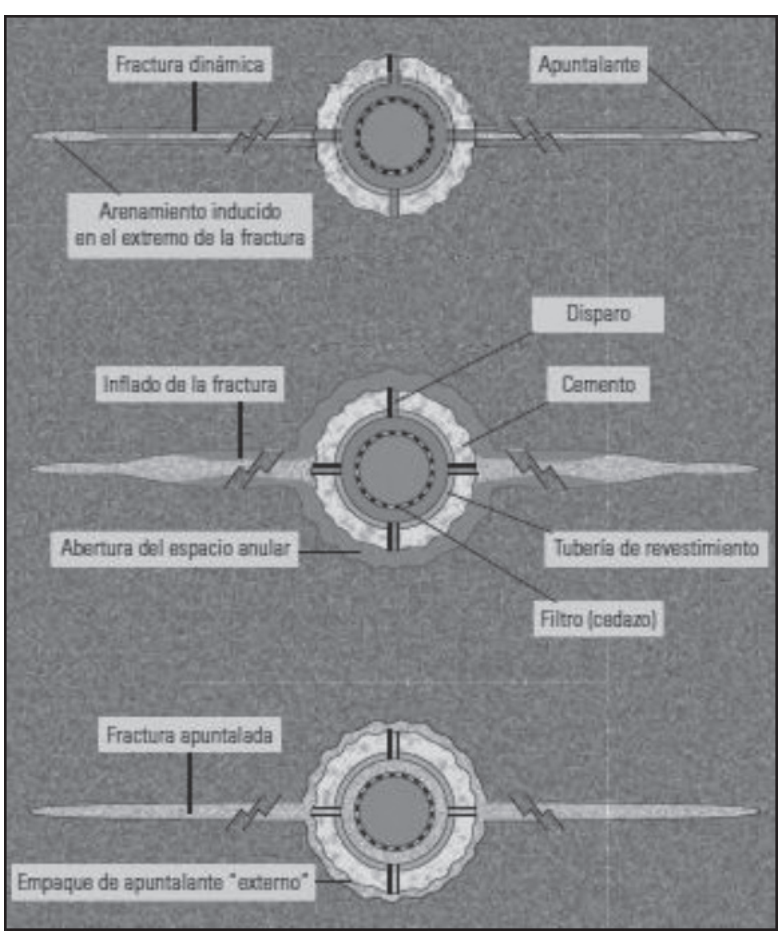

Figura 1. Frac Pack. (Schlumberger, 2012)

Tabla 3. Parámetros de selección del Frac Pack (Norman, 2004), (Schlumberger, 2002), (Monus et al, 1992), (Pandey et al, 2014), (Hainey \& Troncoso, 1992), (Ayoub et al, 1992),

(Bakri, 2008).

\begin{tabular}{|c|c|c|c|}
\hline \multicolumn{2}{|r|}{ Nueva tecnología } & FRAC PACK & Unidades \\
\hline \multicolumn{2}{|r|}{ Tipo de formación } & \begin{tabular}{|c|}
$\begin{array}{c}\text { Arenisca poco consolidada y/o } \\
\text { Altamente susceptible al daño. } \\
(\text { Skin }>2)\end{array}$
\end{tabular} & N/A \\
\hline \multicolumn{2}{|r|}{$\begin{array}{c}\text { Permeabilidad de } \\
\text { formación }\end{array}$} & $<50$ y/0 $100-1.000$ & $\mathbf{m D}$ \\
\hline \multicolumn{2}{|r|}{$\begin{array}{l}\text { Profundidad de } \\
\text { formación TVD }\end{array}$} & $\begin{array}{c}2.000-9.500 \\
\text { (bajo el lecho marino) }\end{array}$ & $\mathbf{F t}$ \\
\hline \multicolumn{2}{|r|}{ Tipo de pozo } & $\begin{array}{c}\text { Horizontal - vertical / simple - } \\
\text { Múltilateral }\end{array}$ & N/A \\
\hline \multicolumn{2}{|c|}{ Presión de yacimiento } & $600-800$ & Psi \\
\hline \multicolumn{2}{|r|}{$\begin{array}{c}\text { Temperatura de } \\
\text { yacimiento }\end{array}$} & $125-300$ & ${ }^{\circ} \mathrm{F}$ \\
\hline \multirow{3}{*}{\multicolumn{2}{|c|}{$\begin{array}{c}\text { Caudal de producción } \\
\text { (por pozo) } \\
\text { Petróleo: 200-700 } \\
\text { Agua: Máx. } 1500\end{array}$}} & Gas: $10-50$ & MMSCFD \\
\hline & & BOPD & \\
\hline & & BWPD & \\
\hline \multicolumn{2}{|r|}{ Erosión } & N/A & $\mathbf{N} / \mathbf{A}$ \\
\hline \multicolumn{2}{|r|}{ Corrosión } & N/A & $\mathbf{N} / \mathbf{A}$ \\
\hline \multirow{4}{*}{ 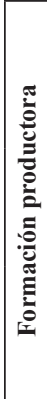 } & $\begin{array}{l}\text { Coeficiente de } \\
\text { uniformidad }\end{array}$ & $5-13$ & Adimensional \\
\hline & \begin{tabular}{|c|} 
Característica \\
(Penberthy y \\
Shaughnessy) \\
\end{tabular} & Altamente no uniforme & N/A \\
\hline & $\mid \begin{array}{c}\text { Rango de } \\
\text { tamaño de grano } \\
\text { (Formación) }\end{array}$ & $50-200$ & Mm \\
\hline & $\begin{array}{c}\text { Clasificación de } \\
\text { rango de tamaño } \\
\text { de grano de arena } \\
\text { (Wentworth) }\end{array}$ & Muy fina - fina & N/A \\
\hline
\end{tabular}




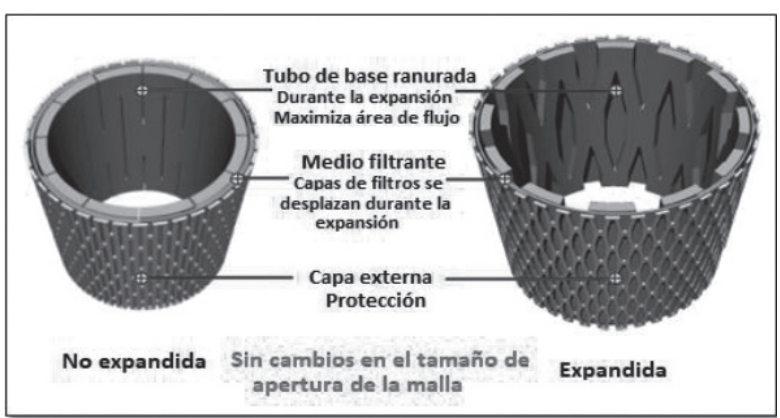

Figura 2. Malla Expandible (MOHD ISMAIL, I. y GEDDES, M). Modificado por los autores.

Tabla 4. Parámetros de selección de la malla Expandible (Salehi-Moorkani \& Ghasemzadeh, 2011), (Weekse et al, 2002), (Mohd \& Geddes, 2013), (Pierfelici et al, 2008).

\begin{tabular}{|c|c|c|c|}
\hline \multicolumn{2}{|r|}{ Nueva tecnología } & MALLA EXPANDIBLE & Unidades \\
\hline \multicolumn{2}{|r|}{ Tipo de formación } & $\begin{array}{c}\text { Areniscas poco } \\
\text { consolidadas. Contenido } \\
\text { de finos } \\
\end{array}$ & N/A \\
\hline \multicolumn{2}{|r|}{$\begin{array}{l}\text { Permeabilidad de } \\
\text { formación }\end{array}$} & $20-1.000$ & mD \\
\hline \multicolumn{2}{|r|}{$\begin{array}{l}\text { Profundidad de } \\
\text { formación TVD }\end{array}$} & \begin{tabular}{|c|}
$\begin{array}{c}4.000-7.000 \\
\text { (bajo el lecho } \\
\text { marino) }\end{array}$ \\
\end{tabular} & $\mathbf{F t}$ \\
\hline \multicolumn{2}{|r|}{ Tipo de pozo } & $\begin{array}{c}\text { Vertical - Desviado } \\
\text { - Horizontal / Simple - } \\
\text { Multilateral } \\
\end{array}$ & N/A \\
\hline \multicolumn{2}{|r|}{ Presión de yacimiento } & $<500$ & Psi \\
\hline \multicolumn{2}{|r|}{$\begin{array}{c}\text { Temperatura de } \\
\text { yacimiento }\end{array}$} & $<225$ & ${ }^{\circ} \mathbf{F}$ \\
\hline \multirow{3}{*}{\multicolumn{2}{|c|}{$\begin{array}{c}\text { Caudal de producción } \\
\text { (por pozo) } \\
\text { Petróleo: Máx. } 30.000 \\
\text { Agua: Máx. } 40.000\end{array}$}} & Gas: Máx. 290 & MMSCFD \\
\hline & & BOPD & \\
\hline & & BWPD & \\
\hline \multirow{2}{*}{\multicolumn{2}{|c|}{ Corrosión }} & \begin{tabular}{|c|} 
Libre de erosión si la \\
velocidad de flujo es menor \\
a 1ft/s en producciones de \\
$2 \mathrm{MMPCD} /$ pozo.
\end{tabular} & N/A \\
\hline & & \begin{tabular}{|c|} 
Resistente a metales UNS \\
N08825, UNS S31254 y \\
UNS N08367 \\
\end{tabular} & N/A \\
\hline \multirow{4}{*}{ 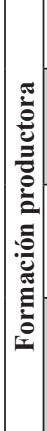 } & $\begin{array}{c}\text { Coeficiente de } \\
\text { uniformidad }\end{array}$ & $2.5-6.7$ & Adimensional \\
\hline & $\begin{array}{l}\text { Característica } \\
\text { (Penberthy y } \\
\text { Shaughnessy) } \\
\end{array}$ & $\begin{array}{c}\text { Uniforme a altamente no } \\
\text { uniforme }\end{array}$ & N/A \\
\hline & \begin{tabular}{|c|} 
Rango de \\
tamaño de grano \\
(Formación)
\end{tabular} & $80-700$ & Mm \\
\hline & \begin{tabular}{|c|}
$\begin{array}{c}\text { Clasificación de } \\
\text { rango de tamaño } \\
\text { de grano de arena } \\
\text { (Wentworth) }\end{array}$ \\
\end{tabular} & Muy fina - gruesa & N/A \\
\hline
\end{tabular}

Malla sinterizada. La malla sinterizada (ver Figura 3 y Tabla 5) consiste en la integración de una tubería base ranurada, barras guía soldadas a esta última y anillos de carburo de silicio instalados sobre las barras guía (Wildhack et al, 2012). Estos anillos poseen separadores entre sí y son mucho más resistentes a la corrosión, la abrasión y la temperatura que los materiales metálicos utilizados en las mallas convencionales (3M, 2017).

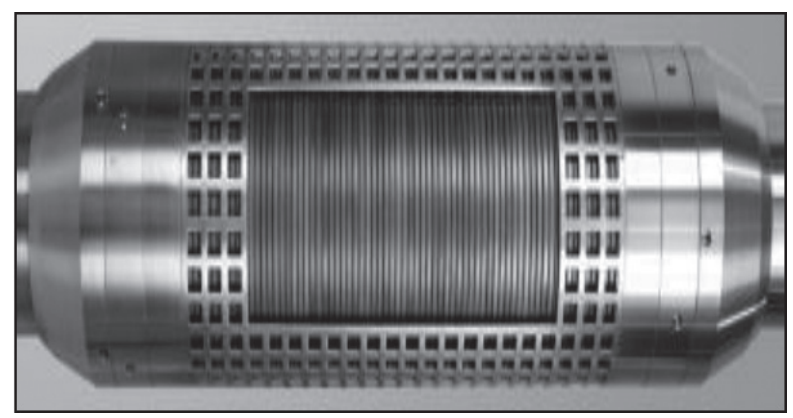

Figura 3. Malla sinterizada (Jackson et al, 2016).

Tabla 5. Parámetros de selección de la malla sinterizada (Wildhack et al, 2012), (3M, 2017), (Müssig et al, 2011), (Nadeem et al, 2014), (Gaurav, 2014), (Jackson et al, 2016), (Risatrio et al, 2015), (Sidek, 2017).

\begin{tabular}{|c|c|c|c|}
\hline \multicolumn{2}{|r|}{ Nueva tecnología } & MALLA SINTERIZADA & Unidades \\
\hline \multicolumn{2}{|r|}{ Tipo de formación } & $\begin{array}{c}\text { Presencia de } \\
\text { intercalaciones de } \\
\text { lutita. Areniscas poco } \\
\text { consolidadas }\end{array}$ & N/A \\
\hline \multicolumn{2}{|r|}{$\begin{array}{l}\text { Permeabilidad de } \\
\text { formación }\end{array}$} & $0,01-550$ & $\mathbf{m D}$ \\
\hline \multicolumn{2}{|r|}{$\begin{array}{l}\text { Profundidad de } \\
\text { formación TVD }\end{array}$} & $3.425-10.400$ & Ft \\
\hline \multicolumn{2}{|r|}{ Tipo de pozo } & $\begin{array}{c}\text { Vertical - Desviado } \\
\text { - Horizontal / Simple - } \\
\text { Multilateral }\end{array}$ & N/A \\
\hline \multicolumn{2}{|r|}{ Presión de yacimiento } & $600-2.250$ & Psi \\
\hline \multicolumn{2}{|r|}{$\begin{array}{l}\text { Temperatura de } \\
\text { yacimiento }\end{array}$} & $180-302$ & ${ }^{\circ} \mathbf{F}$ \\
\hline \multirow{3}{*}{\multicolumn{2}{|c|}{$\begin{array}{l}\text { Caudal de producción } \\
\text { (por pozo) } \\
\text { Petróleo: Máx } 1.400 \\
\text { Agua: Máx } 600\end{array}$}} & Gas: Máx 35 & MMSCFD \\
\hline & & BOPD & \\
\hline & & BWPD & \\
\hline \multirow{2}{*}{\multicolumn{2}{|c|}{ Corrosión }} & \begin{tabular}{|c|} 
Alta resistencia a la \\
erosión contacto con arena \\
de tamaño $20 / 40$ mesh $@$ \\
$30-50 \mathrm{~m} / \mathrm{s}$ durante $60 \mathrm{~min}$ \\
según pruebas. \\
\end{tabular} & N/A \\
\hline & & \begin{tabular}{|c|} 
Alta resistencia a la \\
corrosión en presencia de \\
$\mathrm{HCl}, \mathrm{H} 2 \mathrm{SO} 4$ y salmuera.
\end{tabular} & N/A \\
\hline \multirow{4}{*}{ 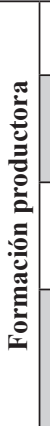 } & $\begin{array}{l}\text { Coeficiente de } \\
\text { uniformidad }\end{array}$ & $2-11$ & Adimensional \\
\hline & $\begin{array}{l}\text { Característica } \\
\text { (Penberthy y } \\
\text { Shaughnessy) } \\
\end{array}$ & $\begin{array}{c}\text { Uniforme a altamente no } \\
\text { uniforme }\end{array}$ & N/A \\
\hline & \begin{tabular}{|c|} 
Rango de \\
tamaño de grano \\
(Formación) \\
\end{tabular} & $10-500$ & Mm \\
\hline & \begin{tabular}{|c|} 
Clasificación de \\
rango de tamaño \\
de grano de arena \\
(Wentworth)
\end{tabular} & Muy fina - media & N/A \\
\hline
\end{tabular}


Malla MeshRite ${ }^{\mathrm{TM}}$. Las mallas MeshRite ${ }^{\mathrm{TM}}$ (ver Figura 4 y Tabla 6) están compuestas por capas comprimidas de lana de acero inoxidable que envuelven una tubería base perforada; a su vez, estas están cubiertas por un velo metálico perforado (Schlumberger, 2017). Además, los poros de la herramienta, que poseen tamaños no uniformes comprendidos entre 15 y 600 micras, permiten el paso de partículas de tamaño menor a 30 micras mientras se ejerce control de arena. La malla MeshRite ${ }^{\mathrm{TM}}$ también presenta insensibilidad a la distribución granulométrica del yacimiento, siendo posible implementarla en formaciones productoras con alto coeficiente de uniformidad o con una distribución granulométrica pobremente definida (Absolute, 2018).

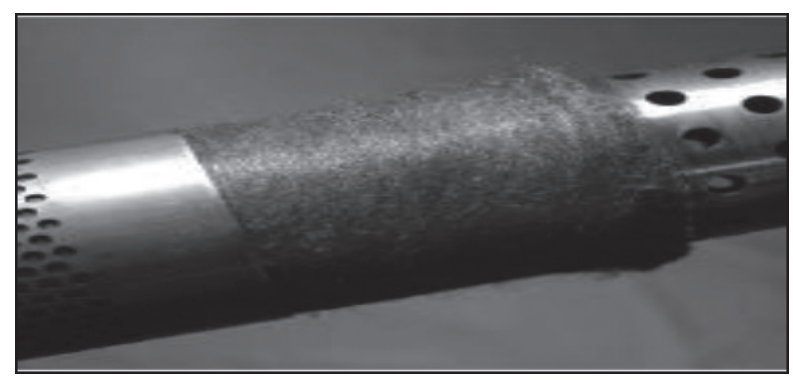

Figura 4. Malla MeshRite ${ }^{\mathrm{TM}}$. (Schlumberger, 2017).

Tabla 6. Parámetros de selección de la malla MeshRiteTM (Schlumberger, 2017), (Sánchez et al, 2004), (Absolute, 2018), (Woiceshyn, 2010), (Huimin, 2011).

\begin{tabular}{|c|c|c|c|}
\hline \multicolumn{2}{|r|}{ Nueva tecnología } & MALLA MESHRITETM & Unidades \\
\hline \multicolumn{2}{|r|}{ Tipo de formación } & $\begin{array}{l}\text { Areniscas poco consolidadas. } \\
\text { Intercalaciones de lutita. }\end{array}$ & N/A \\
\hline \multicolumn{2}{|r|}{$\begin{array}{c}\text { Permeabilidad de } \\
\text { formación }\end{array}$} & $50-3.000$ & mD \\
\hline \multicolumn{2}{|r|}{$\begin{array}{l}\text { Profundidad de } \\
\text { formación TVD }\end{array}$} & $<7.590$ & Ft \\
\hline \multicolumn{2}{|r|}{ Tipo de pozo } & $\begin{array}{c}\text { Vertical - Desviado } \\
\text { - Horizontal / Simple - } \\
\text { Multilateral }\end{array}$ & N/A \\
\hline \multicolumn{2}{|c|}{\begin{tabular}{|l|} 
Presión de yacimiento \\
\end{tabular}} & $<3.000$ & psi \\
\hline \multicolumn{2}{|c|}{\begin{tabular}{|c|}
$\begin{array}{c}\text { Temperatura de } \\
\text { yacimiento }\end{array}$ \\
\end{tabular}} & $<\mathbf{2 0 0}$ & ${ }^{\circ} \mathbf{F}$ \\
\hline \multirow{3}{*}{\multicolumn{2}{|c|}{$\begin{array}{l}\text { Caudal de producción } \\
\text { (por pozo) } \\
\text { Petróleo: Máx. 1.070. } \\
\text { Agua: Máx. 1.000. }\end{array}$}} & Gas: Máx. 4,5 & MMSCFD \\
\hline & & BOPD & \\
\hline & & BWPD & \\
\hline \multicolumn{2}{|r|}{ Erosión } & $\begin{array}{c}\text { Mejor resistencia a la erosión } \\
\text { de malla de espesor de } 19 \\
\mathrm{~mm} \text { vs espesor de } 9,5 \mathrm{~mm} \text {. }\end{array}$ & N/A \\
\hline & Corrosión & Resistente a $\mathrm{H} 2 \mathrm{~S}$ y $\mathrm{CO} 2$ & N/A \\
\hline \multirow{4}{*}{ 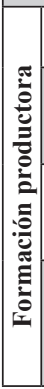 } & $\begin{array}{c}\text { Coeficiente de } \\
\text { uniformidad }\end{array}$ & $2-12$ & Adimensional \\
\hline & \begin{tabular}{|c|} 
Característica \\
(Penberthy y \\
Shaughnessy)
\end{tabular} & $\begin{array}{c}\text { Uniforme a altamente no } \\
\text { uniforme }\end{array}$ & N/A \\
\hline & \begin{tabular}{|c} 
Rango de \\
tamaño de grano \\
(Formación)
\end{tabular} & $35-250$ & $\mu \mathrm{m}$ \\
\hline & \begin{tabular}{|c|}
$\begin{array}{c}\text { Clasificación de } \\
\text { rango de tamaño } \\
\text { de grano de arena } \\
\text { (Wentworth) }\end{array}$ \\
\end{tabular} & Muy fina-Fina & N/A \\
\hline
\end{tabular}

Matriz de decisión para tecnologías nuevas y convencionales de control de arena. Teniendo en cuenta los parámetros de operación de las Tablas 3, 4,5 y 6 , se procede a elaborar una matriz de decisión para presentar el contraste entre las tecnologías nuevas a implementar al MGCP y las ya utilizadas, sirviendo así como una herramienta para la recomendación de tecnologías nuevas y convencionales. (ver Tabla 7)

Tabla 7. Matriz de decisión entre las tecnologías nuevas y convencionales (Beltrán \& Carvajal, 2018).

\begin{tabular}{|c|c|c|c|c|c|}
\hline & Tecnología & $\begin{array}{c}\text { Tipo de } \\
\text { arena } \\
\text { (yacimiento) }\end{array}$ & $\begin{array}{c}\text { Coeficiente } \\
\text { uniformidad } \\
\text { yacimiento } \\
\text { (U) }\end{array}$ & $\begin{array}{c}\text { Característica } \\
\text { (yacimiento) }\end{array}$ & $\begin{array}{c}\text { Rango de } \\
\text { tamaño } \\
\text { grano en } \\
\text { yacimiento } \\
\text { (micras) }\end{array}$ \\
\hline \multirow{4}{*}{ 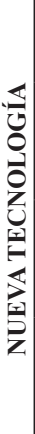 } & Frac pack & $\begin{array}{c}\text { Muy } \\
\text { fina- fina }\end{array}$ & $5-13$ & $\begin{array}{c}\text { Altamente no } \\
\text { uniforme }\end{array}$ & $50-250$ \\
\hline & $\begin{array}{c}\text { Malla } \\
\text { expandible }\end{array}$ & $\begin{array}{c}\text { Muy } \\
\text { fina-gruesa }\end{array}$ & $2,5-6,7$ & $\begin{array}{l}\text { Uniforme a } \\
\text { altamente no } \\
\text { uniforme }\end{array}$ & $80-700$ \\
\hline & $\begin{array}{c}\text { Malla } \\
\text { sinterizada }\end{array}$ & $\begin{array}{c}\text { Muy } \\
\text { fina-media }\end{array}$ & $2-11$ & $\begin{array}{l}\text { Uniforme a } \\
\text { altamente no } \\
\text { uniforme }\end{array}$ & $10-500$ \\
\hline & MeshRite $^{\mathrm{TM}}$ & $\begin{array}{c}\text { Muy } \\
\text { fina-fina }\end{array}$ & $2-12$ & $\begin{array}{l}\text { Uniforme a } \\
\text { altamente no } \\
\text { uniforme }\end{array}$ & $35-250$ \\
\hline \multirow{4}{*}{ 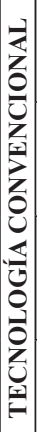 } & $\begin{array}{c}\text { Liner } \\
\text { ranurado }\end{array}$ & $\begin{array}{c}\text { Muy } \\
\text { fina-gruesa }\end{array}$ & $<1,5$ & Uniforme & $62-1000$ \\
\hline & $\begin{array}{c}\text { Malla } \\
\text { convencional }\end{array}$ & Fina-Media & $<3$ & Uniforme & $126-500$ \\
\hline & $\begin{array}{c}\text { Malla } \\
\text { premium }\end{array}$ & Media & $<5$ & $\begin{array}{c}\text { Uniforme a no } \\
\text { uniforme }\end{array}$ & $251-500$ \\
\hline & Gravel pack & Fina-gruesa & $<5$ & $\begin{array}{c}\text { Uniforme a no } \\
\text { uniforme }\end{array}$ & $126-1000$ \\
\hline
\end{tabular}

De acuerdo con la tabla anterior, las cuatro nuevas tecnologías de control de arena restringen la producción de granos provenientes de formaciones que posean una característica altamente no uniforme, en comparación con las herramientas convencionales, reteniéndose así granos de menor tamaño.

Adicionalmente, las nuevas tecnologías de control de arena permiten ejercer dicha función en formaciones con valores mínimos de rango de tamaño de grano hasta 62 micras, siendo este valor el mínimo aplicable para las tecnologías convencionales. El valor mínimo de dicho parámetro aplicable para las nuevas tecnologías es equivalente a 10 micras, el cual corresponde a la malla sinterizada. 
3. Matriz teórica para los campos del estudio (herramienta 1). Tal y como se explicó anteriormente, esta matriz fue complementada mediante la incorporación de las nuevas tecnologías gracias al uso de la anterior matriz de decisión. El modo de uso de la herramienta se encuentra explicado la Figura 5.

Identificación de los métodos convencionales de control de arena en los campos y problemas de arenamiento de estos últimos. En esta sección se explicará el procedimiento para identificar el método de control de arena más usado en los pozos de estudio y los campos más críticos en materia de arenamiento. Para su realización, se llevaron a cabo distribuciones porcentuales de los mecanismos convencionales de control de arena instalados en los 24 campos de estudio y de los yacimientos estudiados (González \& Ramírez, 2016) según su coeficiente de uniformidad y rango de tamaño de grano, tomándose como base el primero de estos últimos dos parámetros. Así mismo, se determinaron los problemas de producción de arena para cada campo mediante la elaboración de una base de datos compuesta por más de treinta artículos académicos y trabajos de grado con el fin de complementar la distribución porcentual (Beltrán \& Carvajal, 2018).

Las distribuciones porcentuales y la identificación de los problemas de arenamiento se llevaron a cabo para cada campo puesto que González y Ramírez asumieron que sus respectivos pozos producían de una misma formación teniendo en cuenta que los métodos convencionales de control de arena instalados poseían características muy similares que permitieron calcular un tamaño de grano común según los criterios de selección de tamaño de grava utilizados (González \& Ramírez, 2016).

4. Herramienta de selección de mecanismos de control de arena (herramienta 2). La figura 6 presenta el procedimiento realizado para la recomendación de una tecnología mecánica en función del coeficiente de uniformidad y el tamaño de grano promedio a partir de dos datos de entrada como el tamaño de grano de la arena de la formación productora o producida y la frecuencia de estos. Además de realizar un cálculo comparativo utilizando las ecuaciones $2,3,4$ y 5 para determinar la configuración de la ranura en las mallas o liner y el tamaño de grava comercial para el caso del frac pack o grave pack. (ver figura 6 en Anexos).

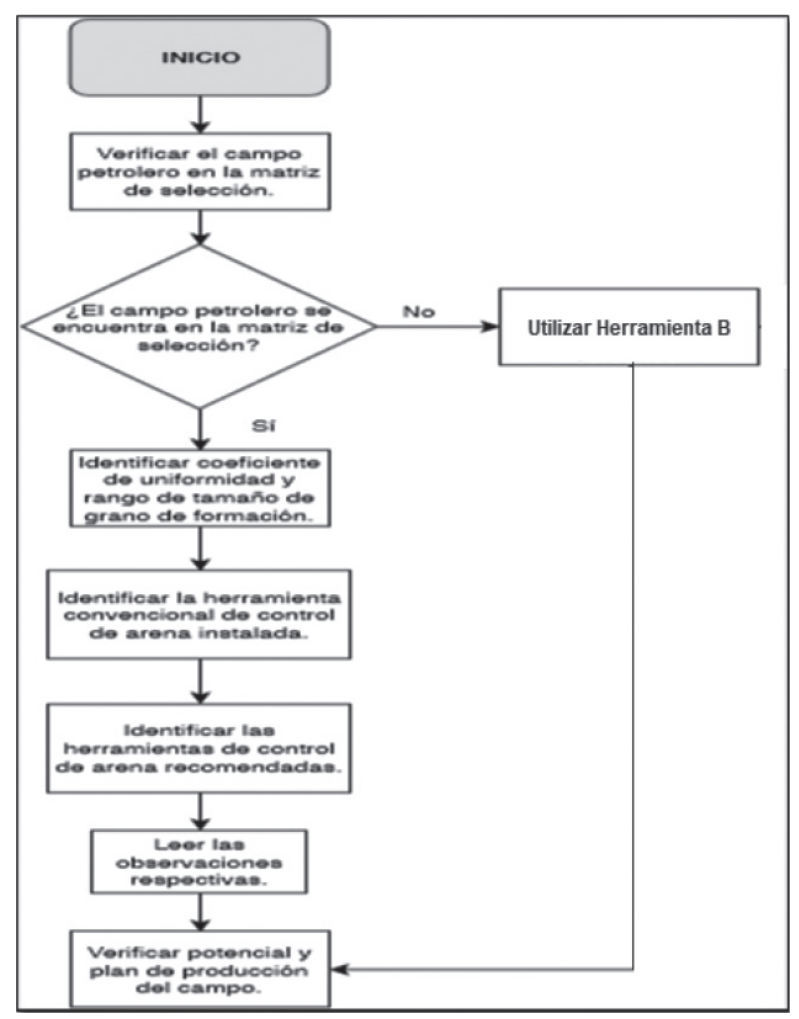

Figura 5. Procedimiento de utilización de la herramienta 1 de selección de herramientas mecánicas de control de arena (González \& Ramírez, 2016). Modificado por los autores.

\section{Resultados}

\section{Identificación de los métodos convencionales de control de arena en los campos y problemas de arenamiento de estos últimos}

Para determinar los campos más críticos en problema de arenamiento se realizó, en primer lugar, una distribución porcentual de los campos con el fin de identificar aquellos cuyos yacimientos se encuentran caracterizados como altamente no uniformes según sus coeficientes de uniformidad. Acto seguido, se realiza un diagrama de torta de este último grupo de campos identificados para determinar aquellos cuyos reservorios presentan rangos de tamaños de grano de arena finos y muy finos en virtud de la granulometría del yacimiento como tal. Por último, se utilizó la base de datos mencionada anteriormente para determinar el número de problemas generales de producción de arena presentados en cada campo, robusteciendo así la distribución porcentual.

Distribución porcentual de los métodos de control de arena de tipo mecánico instalados originalmente. González y Ramírez elaboraron una distribución porcentual total de los mecanismos de control de 
arena instalados en los 116 pozos que fueron objeto de análisis durante la elaboración del trabajo de grado correspondiente (González \& Ramírez, 2016). Según esta distribución porcentual, el liner ranurado es la herramienta de control mecánico de arena convencional más utilizada en la muestra estudiada. De un total de 116 pozos, este método de control de arena fue instalado en 91 pozos. No obstante, el liner ranurado fue utilizado como única herramienta de control de arena en 65 pozos mientras que fue implementado junto con el empaquetamiento con grava en 26 pozos. Lo anterior se encuentra representado mediante la Figura 7.

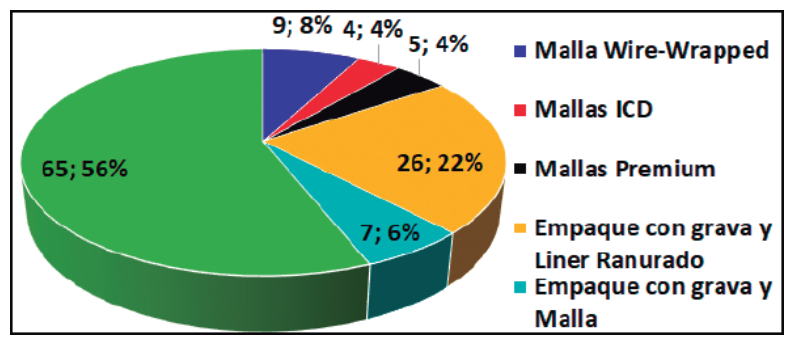

Figura 7. Distribución porcentual total de las herramientas mecánicas de control de arena instaladas (González \& Ramírez, 2016). Modificado por los autores.

Identificación de los campos petrolíferos de estudio más críticos en materia de producción de arena. A continuación, se identifican los campos más críticos en materia de arenamiento según las características granulométricas y problemas de producción de arena presentados en sus yacimientos.

Distribución porcentual de los coeficientes de uniformidad correspondientes a las formaciones productoras de los campos petrolíferos de estudio. Según la Figura 8, aproximadamente, el 71\% de los campos de estudio de la fase 1 del proyecto presenta formaciones productoras con característica altamente no uniforme. Dentro de este grupo se encuentran los campos que poseen los problemas más críticos de producción de arena dado que las tecnologías convencionales poseen rangos de operación recomendadas para coeficientes de uniformidad menores a 5. La malla convencional y la malla premium son recomendadas para ser empleadas en formaciones productoras cuyos coeficientes de uniformidad sean menores a 3 y a 5 respectivamente (Bellarby, 2009). Además, los liner ranurados deben usarse en formaciones uniformes que posean coeficientes de uniformidad menores a 1,5 (Penberthy \& Shaughnessy, 1992). Así mismo, puede implementarse el empaquetamiento con grava para formaciones con coeficientes de uniformidad menores a 5 y emplear el criterio de Saucier para la selección del tamaño de la grava (Soler \& Suárez, 1991).

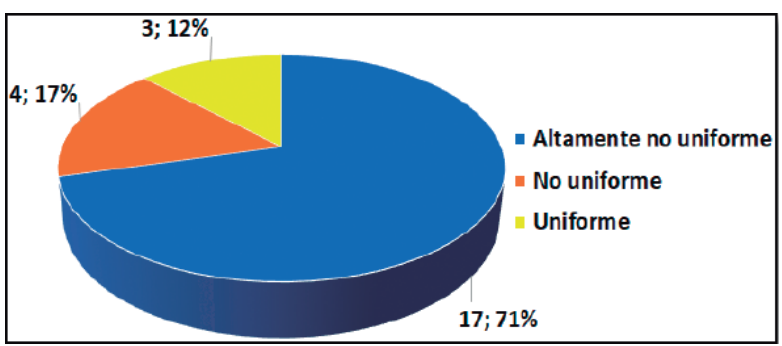

Figura 8. Distribución porcentual de la característica de uniformidad de los yacimientos correspondientes a la muestra de veinticuatro campos de estudio según su coeficiente de uniformidad (González \& Ramírez, 2016). Modificado por los autores.

Distribución porcentual de las formaciones
productoras altamente no uniformes correspondientes a los veinticuatro campos de estudio, según sus rangos de tamaño de grano para identificación de campos problemáticos. Según la Figura 9, aproximadamente seis de los diecisiete campos que presentan areniscas altamente no uniformes poseen un rango de tamaños de grano de arena comprendido entre 126 y 250 micras, el cual está clasificado como tamaño de grano fino; entre tanto, solo un campo presenta rango de tamaño de arena muy fino, el cual está comprendido entre 62 y 125 micras.

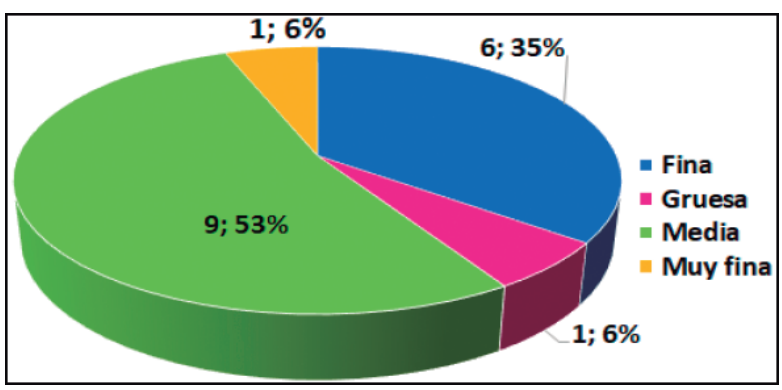

Figura 9. Distribución porcentual de los tamaños de arena presentes en los campos con areniscas altamente no uniformes (González \& Ramírez, 2016). Modificado por los autores.

Empleo de la base de datos teórica en la determinación de los campos más problemáticos en materia de arenamiento. Teniendo en cuenta la distribución porcentual anteriormente realizada y la base de datos utilizada por Beltrán y Carvajal (2018), se identificó que en los siete campos identificados mediante las distribuciones porcentuales anteriores predomina la presentación de problemas de arenamiento relacionados a la 
migración de granos finos y taponamiento en los cañoneos y ranuras de los mecanismos de control de arena de tipo mecánico utilizados. Además, se determinó que los campos más problemáticos en materia de arenamiento se localizan en la cuenca del Valle Medio del Magdalena, presentando como problemas comunes la ocurrencia de taponamientos en las líneas de superficie, migración de granos finos, fallas en los sistemas de levamiento artificial y averías en las tuberías de revestimiento (Beltrán $\&$ Carvajal, 2018). (Ver Figura 10 en Anexos).

\section{Matriz teórica para los campos del estudio}

Las nuevas tecnologías de control de arena de tipo mecánico pueden ser recomendadas para uso en formaciones con coeficientes de uniformidad mayores a cinco y rangos de tamaño de arena finos y muy finos. Por lo tanto, las nuevas herramientas fueron recomendadas paras su uso en los yacimientos presentes en 22 de los 24 campos estudiados, siendo la malla sinterizada la más formulada para su uso en 21 de los 24 campos de estudio.

Así mismo, la inclusión de nuevas tecnologías permitió recomendar una tecnología a 11 de los 13 campos para los cuales previamente no era posible recomendar una tecnología convencional debido a un máximo control de partículas con coeficiente de uniformidad de 5 y recomendar una más precisa para los demás campos ajustándose a los problemas presentados. (Ver Figura 11 en Anexos.)

Herramienta de selección de mecanismos de control de arena. (herramienta 2) La presente plataforma consta de tres pasos: Ingreso de datos, corrida del programa y recomendación de la configuración de una tecnología de control. Inicialmente, la plataforma requiere el ingreso manual o la importación de datos desde una tabla en Excel. Para el primer caso, solicita el ingreso de los tamaños de grano (62 a 2000 micrasclasificación de Wentworth) (Jackson \& Richardson, 2007), y su peso, además de un nombre a la muestra. El programa realiza los cálculos, y presenta una tabla llamada "datos granulométricos de la muestra de interés" con los datos previamente ingresados, "la curva de distribución granulométrica" construida, de la cual determina los percentiles, P10 (X90), P40 (X60), P50 (X50), P70 (X30) y P90 (X10), los cuales son presentados en la tabla "variables granulométricas calculadas" utilizadas para la selección y diseño de mecanismos de control de arena mostrados en la tabla "mecanismos de control de arena sugeridos" donde para el caso de recomendar el frac pack o gravel pack también presenta el tamaño de la grava.

Por último, la página presenta la tabla "Recomendaciones para la configuración / Diseño del mecanismo de control de arena" para el caso único donde se recomiendo el liner ranurado cualquier tipo de malla, basado en el cálculo comparativo entre 4 autores. (Ver Tablas 8, 9, 10, 11 y Figura 12 en Anexos).

\section{Discusión}

En este estudio se pudo demostrar como la ampliación del coeficiente de uniformidad y la minimización en el tamaño de grano promedio mediante la implementación de nuevas tecnologías a las dos herramientas del MGCP satisfago en primera instancia, los parámetros granulométricos en 11 de los 13 campos donde previamente no era posible el uso de tecnologías para ejercer el control de arena. En segunda instancia, proporcionó un grado de exactitud mayor al dado únicamente por las convencionales. En este sentido, el módulo de control de arena aporta al ámbito educativo como una herramienta complementaria de aprendizaje atribuyendo posibles soluciones bajo condiciones reales de la industria. Sin embargo, es necesario robustecer y actualizar algunos parámetros mediante un análisis de factibilidad de las tecnologías y una actualización del coeficiente de uniformidad como parámetro de selección.

\section{Conclusiones}

- El liner ranurado es la tecnología convencional de control de arena más utilizada, siendo aplicada en 65 de los 116 pozos. Se identificó a la cuenca del Valle Medio del Magdalena como la más problemática en arenamiento entre las cinco estudiadas.

- Las cuatro nuevas tecnologías restringen la producción de arena en formaciones con distribución granulométrica altamente no uniforme y tamaños de grano cada vez menores en comparación a las herramientas convencionales.

- La matriz teórica de selección complementada ayudó a recomendar el uso de tecnologías de control de arena a 22 de los 24 campos estudiados. Así mismo, las nuevas tecnologías fueron recomendadas a 11 de los 13 campos para los cuales no era posible sugerir el uso de una herramienta.

- La actualización de la herramienta 2 aportó un incremento en el grado de confiabilidad debido a la incorporación de un segundo parámetro de selección 
(tamaño de grano promedio) en conjunto con el ya aplicado coeficiente de uniformidad, además de ser una herramienta aplicable a datos granulométricos de la arena de formación o producida.

- El módulo General de Completamiento de pozos puede ser una herramienta complemento de aprendizaje en áreas tales como perforación, producción y yacimientos proporcionando soluciones bajo condiciones reales de producción.

\section{Recomendaciones}

- Incluir costos de las tecnologías en la matriz de selección para de esta forma realizar un análisis de factibilidad de la aplicabilidad de las herramientas y se robustezca el MGCP.

- Debido a que el coeficiente de uniformidad es un parámetro muy antiguo de granulometría es posible estudiar nuevos parámetros de selección de herramientas mecánicas de control de arena para su implementación en el MGCP desde el año 2000 hasta la actualidad.

- Estudiar el criterio de Tiffin (1998) para la determinación del tamaño de grava dado que emplea más parámetros de la granulometría del yacimiento para así buscar una mayor recuperación de hidrocarburos de acuerdo con la ecuación 7.

$\frac{d 10}{d 95}<10, \frac{d 40}{d 90}<5$, sub 325 mesh $<5 \% \rightarrow 7 \sim 8 * d 50$

A continuación, se indican las variables de la anterior ecuación:

$\mathrm{d} 10=$ Percentil 10 de la distribución granulométrica del yacimiento.

d40= Percentil 40 de la distribución granulométrica del yacimiento.

d50= Percentil 50 de la distribución granulométrica del yacimiento.

d90= Percentil 90 de la distribución granulométrica del yacimiento.

d95= Percentil 95 de la distribución granulométrica del yacimiento.

Sub 325 mesh $=$ Porcentaje de partículas de tamaño inferior a 44 micras.

\section{Agradecimientos}

Agradecemos a la Fundación Universidad de América, por permitir nuestra formación como ingenieros de petróleos y elaborar el trabajo de grado del cual se derivó el presente artículo. Así mismo, agradecemos especialmente al ingeniero Andrés Felipe Suárez Barbosa -director de dicho trabajo de grado-, Eliana Mesa y el geólogo Franklin Yoris por habernos brindado su apoyo, orientación académica y confianza para desarrollar este proyecto satisfactoriamente. Adicionalmente, agradecemos a Kevin Peña, uno de los creadores del Manual General de Completamiento de pozos (MGCP), quien nos brindó su apoyo fundamental en la incorporación de las mejoras desarrolladas al módulo de control de arena del aplicativo web mediante este proyecto.

\section{Referencias}

1. 3M. (2017). 3M Ceramic Sand Screens, pp. 1-2. Obtenido de: https://multimedia.3m.com/mws/ media/1382044O/ceramic-sand-technicaldata-sheet.pdf

2. Absolute. (2018). MeshRite ${ }^{\mathrm{TM}}$ Brochure. Obtenido de: http://product.absolutect.com/meshritebrochure.

3. Ayoub, J.A., Kirksey, J.M., Malone, B.P. and Norman W.D. (1992). Hydaulic Fracturing of Soft Formations in the Gulf Coast, SPE Formation Damage Control Symposium, SPE-23777-MS, Lafayette, Louisiana, USA.

4. Bakri, M, et al. (2008). A Case Study in the Successful Design and Implementation of Frac-Pack Treatments in a Challenging Workover Environment in Malaysia. SPE Asia Pacific Oil and Gas Conference and Exhibition, SPE116913-MS, Perth, Australia.

5. Ballesteros, R. A. M., \& González, F. E. C. (2016). Modelado del efecto de la movilidad del banco de finos de propante sobre las tendencias de producción en pozos hidráulicamente fracturados. Revista Fuentes, 14(2), 41-49.

6. Bellarby, J. (2009). Well Completion Design, Oxford, Reino Unido: Elsevier, pp. 162, 172.

7. Beltrán, G.A. and Carvajal, J.S. (2018). Generación de un programa informático mejorado para la selección de nuevas tecnologías de control de arena de tipo mecánico en Ecopetrol S.A. Bogotá, D.C., Colombia: Fundación Universidad de América, pp. 37, 38, 42, 47, 48, 49, 56.

8. Gaurav, K. et al. (2014). An Innovative Approach of Revival for Damaged Wells in High Erosive Environment using Ceramic Sand Screens. Offshore Technology Conference, OTC 25106MS, Houston, Texas, USA.

9. González, E.F. and Ramírez, J.D. (2016). Diseño de una metodología de selección preliminar de sistemas de control de arena para los 
campos operados por Ecopetrol S.A. en Colombia. Bogotá, D.C., Colombia Fundación Universidad de América, pp. 54, 119.

10. Guardia, V. M. D., Torres, M. C., Arenas, C. E. V., Castro, R. H., Toro, G. M., \& Mendoza, O. B. (2011). Análisis de riesgo y simulación de monte carlo en la valoración de proyectosaplicación en la industria de los hidrocarburos. Revista Fuentes, 9(2).

11. Hainey, B. W. y Troncoso, J. C. (1992). Frac-Pack: An Innovative Stimulation and Sand Control Technique, SPE Formation Damage Control Symposium, SPE-23777-MS, Lafayette, Louisiana, United States of America.

12. Huimin, Ye, et al. (2011). Innovative WellCompletion Strategy for Challenging HeavyOil Wells within Mature Fields Requiring Sand Control in Colombia, SPE Heavy Oil Conference and Exhibition, SPE 149966-MS Kuwait City, Kuwait.

13. Jackson, S. R.; Gundemoni, B. and Barth, P. (2016). Sand Control in Corrosive and Erosive Downhole Conditions at High Temperatures, SPE Asia Pacific Oil \& Gas Conference and Exhibition, SPE 182278-MS Perth.

14. Jackson, D.R. and Richardson, M.D. (2007). HighFrequency Seafloor Acoustics. NewYork, USA: Springer, pp 78, 79.

15. Mohd Ismail, I. y Geddes, M. W. (2013). Fifteen Years of Expandable Sand Screen Performance and Reliability, SPE Annual Technical Conference and Exhibition, SPE-166425-MS, New Orleans, Louisiana, USA.

16. Monus F.L., Broussard F.W., Ayoub J.A. y Norman W.D. (1992). Fracturing Unconsolidated Sand Formations Offshore Gulf of Mexico, SPE Annual Technical Conference and Exhibition, SPE-24844-MS, Washington, D.C., USA.

17. Müssig, S. et al. (2011). Ceramic Screens, an Innovative Milestone in Sand Control, SPE Annual Technical Conference and Exhibition, SPE 146721, Denver, Colorado, USA.

18. Nadeem, A., Lopez, M., Joly, S. et al. (2014). Ceramic Screens - an Innovative Downhole Sand Control Solution for Old and Challenging Cased Hole Completions, International Petroleum Technology Conference, IPTC 17477, Doha, Qatar.

19. Norman, D. (2004). The Frac-Pack Completion: Why has it Become the Standard Strategy for Sand Control? Obtenido de: https:// Www.onepetro.org/general/SPE-101511DL? sort $=\&$ start $=0 \& q=$ The + Frac-Pack + Compl etion $\% 3 \mathrm{~A}+$ Why + has $+\mathrm{it}+\mathrm{Become}+$ the + Standar $\mathrm{d}+$ Strategy+for + Sand + Control $\% 3 \mathrm{~F}++\&$ from 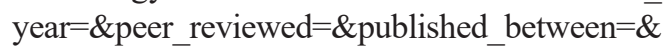 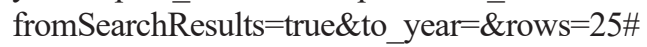

20. Páez, E. G. M., González, F. E. C., \& Duarte, C. A. M. (2016). Aplicación de series de tiempo en la realización de pronósticos de producción. Fuentes: El reventón energético, 14(1), 79-88.

21. Pandey, V.J.; Burton, R.C. y Nozaki, M. (2014). Evolution of Frac-Pack Design and Completion Procedures for High Permeability Gas Wells in Subsea Service, Society of Petroleum Engineers, obtenido de: https://doi. org/10.2118/168636-PA.

22. Penberthy JR, W.L. and Shaughnessy, C.M. (1992). Sand Control, USA: SPE series on special topics, pp. 20, 21, 24, 32.

23. Pierfelici, S., Tacconelli, A., Ripa, G. et al. (2008). High Gas Rate Production: Expandable Solutions in the Mediterranean Sea, SPE Annual Technical Conference and Exhibition, SPE-115860-MS, Denver, Colorado, USA.

24. Quintero, Y. A., Triana, R. L., Jaimes, M. G., \& Torres, M. L. (2010). Optimización de diseños de fractura hidráulica aplicando estudios geomecánicos. Revista fuentes, 8(2).

25. Risatrio, A. A., et al. (2015). Cased Hole Ceramic Screen Cutting Completion Cost for Marginal Reservoir: Application in Tunu Field, SPE/ ATMI Asia Pacific Oil \& Gas Conference and Exhibition, SPE 176225-MS, Nus Dua, Bali, Indonesia.

26. Salehi-Moorkani, R. and Ghasemzadeh, A. (2011). Improvement of the Criteria for Expandable Sand Screen Well Candidate Selection, SPE European Formation Damage Conference, SPE-143377MS, Noordwijk, The Netherlands.

27.Sanchez, H., Sampedro, T. and Peñaranda, R. (2004). An Efficient Alternative to Control Sand Production in Horizontal Wells in Block-16 of Ecuador, Offshore Technology Conference, OTC 16256 Houston, Texas, USA.

28. Saucier, R.J. (1974). Considerations in Gravel Pack Design, SPE-AIME 47 $7^{T H}$ Annual Fall Meeting, SPE 4030, San Antonio, Texas, USA.

29. Schlumberger. (2002). Método combinado de estimulación y control de la producción de arena, Oilfield review, pp. 32-53. Obtenido de: https://www.slb.com/ /media/Files/resources/ oilfield_review/spanish02/aut02/p32_53.pdf

30. Schlumberger. (2017). MeshRite ${ }^{\mathrm{TM}}$. Obtenido de: http://www.slb.com/ /media/Files/sand control/product_sheets/MeshRite_screens_ 
ps.pdf

31. Sidek, S., et al. (2017). First Successful Application of Ceramic Sand Screen in Maturing Oil Field, Offshore East Malaysia, Abu Dhabi International Petroleum Exhibition \& Conference, SPE 188537-MS Abu Dhabi, UAE.

32. Soler, F. and Suárez, C.R. (1991). Técnicas y procedimientos utilizados para el control de la producción de arena en pozos horizontales $y$ verticales, Bogotá, D.C., Colombia: Fundación Universidad de América, pp. 35.

33. Tamayo, G. A. V., Consuegra, F. R., \& Simancas, M. E. C. (2017). Predicción de flujo multifásico en sistemas de recolección de crudo: descripción de requerimientos. Fuentes: El reventón energético, 15(1), 87-99. 29. TIFFIN, D.L. et al. (1998). New Criteria for Gravel and Screen Selection for Sand Control, SPE Formation Damage Control Conference, SPE 39437, Lafayette, LA, USA.
34. Weekse, A., Steve, G. and Urselmann, R. (2002). Expandable Sand Screen: Three New World Records in the Brigantine Field, IADC/SPE Drilling Conference, SPE-74549-MS Dallas, Texas, USA.

35. Wildhack, S., Lesniak, C., Müssig, S. et al. (2012). Ceramic Sand Screens for Sand Control in Unconsolidated Reservoirs: Application and Sand Retention Capabilities in the Maturing Oil Field, Gaiselberg, Austria, SPE Annual Technical Conference and Exhibition, SPE 160327 San Antonio, Texas, USA.

36. Woiceshyn, G. and Russel, T. A Unique Sand Control Screen that Enhances Productivity, SPE Latin American and Caribbean Petroleum Engineering Conference, SPE 139360, Lima, Peru.

37. Xiang, W. (2003). Application of Bridging theory on Saucier gravel to examine the sand control effect. SPE Asia Pacific Oil and Gas Conference and Exhibition, SPE 80450, Jakarta, Indonesia.

Recepción: 17 de octubre de 2018

Aceptación: 03 de mayo de 2019 


\section{Anexos}

Procedimiento de utilización de la herramienta 2 de selección de herramientas mecánicas de control de arena.

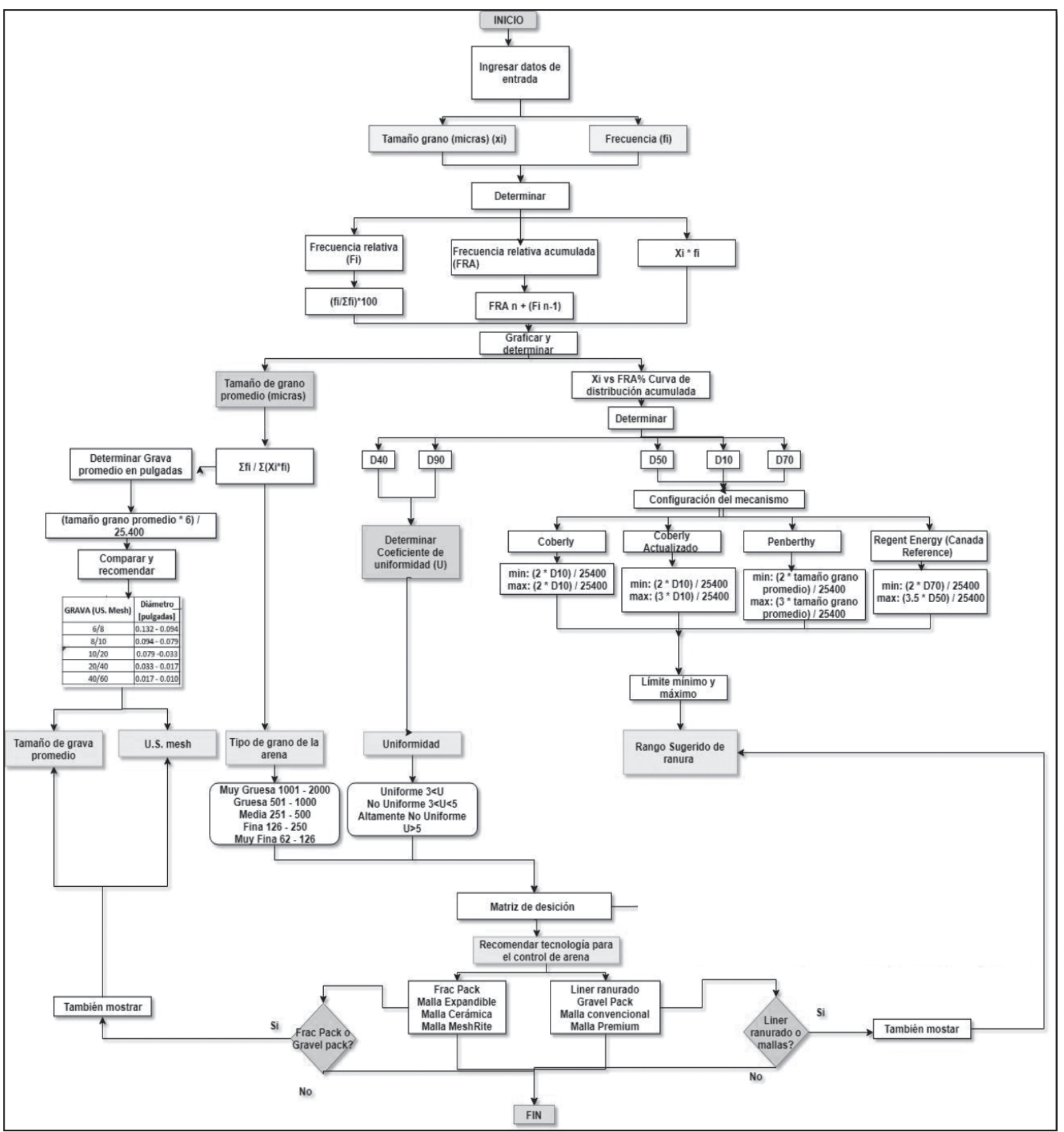

Figura 6. Procedimiento de utilización de la herramienta 2 de selección de herramientas mecánicas de control de arena. (Beltrán \& Carvajal, 2018).

Campos más críticos en materia de arenamiento según sus respectivos problemas generales de producción de arena 


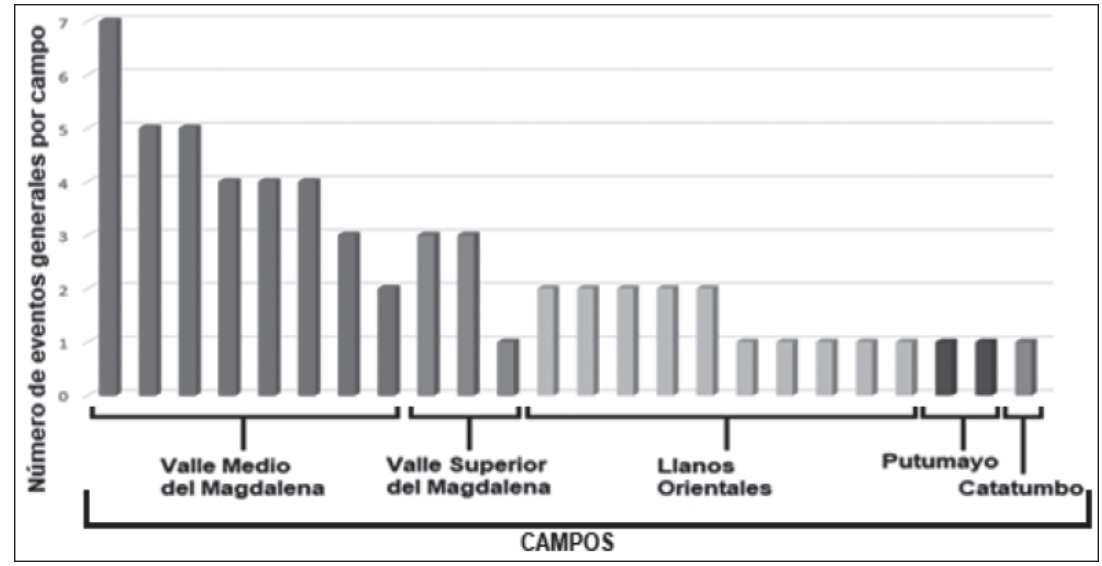

Figura 10. Campos más críticos en materia de arenamiento según sus respectivos problemas generales de producción de arena (Beltrán \& Carvajal, 2018).

Frecuencia de recomendación de las herramientas mecánicas de control de arena.

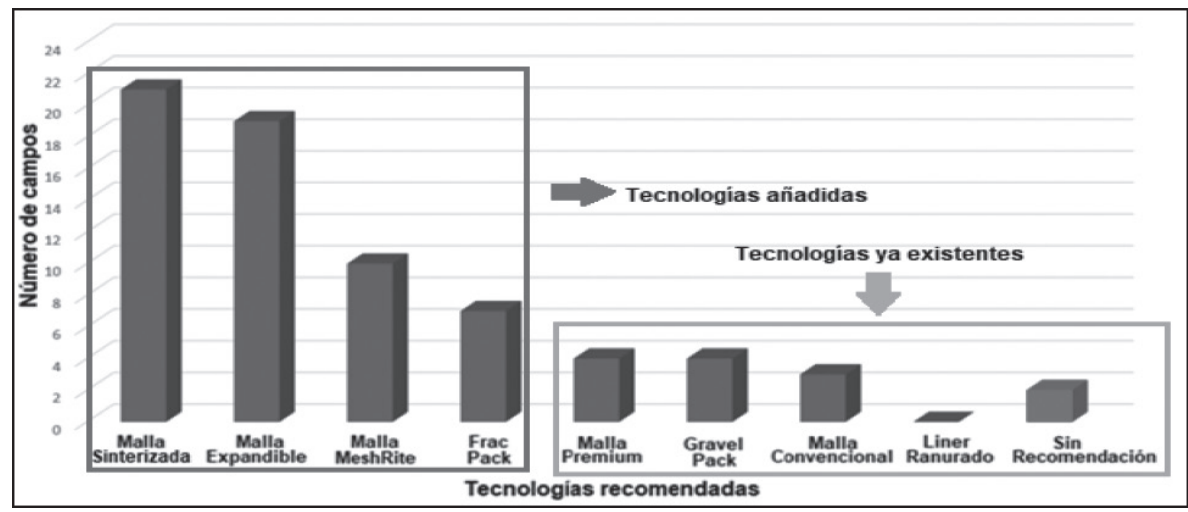

Figura 11. Frecuencia de recomendación de las herramientas mecánicas de control de arena (González \& Ramírez, 2016). Modificado por los autores.

Herramienta de selección mecanismos de control de arena.

Tabla 8. Datos granulométricos de la muestra de interés. (Beltrán \& Carvajal, 2018).

\begin{tabular}{|c|c|}
\hline \multicolumn{2}{|c|}{ Datos granulométricos de la muestra de interés } \\
\hline Tamaño de grano (Micras) & Peso de muestra (gr) \\
\hline 64 & 10 \\
\hline 65 & 5 \\
\hline 65 & 20 \\
\hline 66 & 30 \\
\hline 67 & 45 \\
\hline 68 & 60 \\
\hline 69 & 80 \\
\hline 270 & 34 \\
\hline 300 & 56 \\
\hline 350 & 67 \\
\hline 400 & 90 \\
\hline 500 & 234 \\
\hline 600 & 300 \\
\hline 1000 & 24 \\
\hline
\end{tabular}




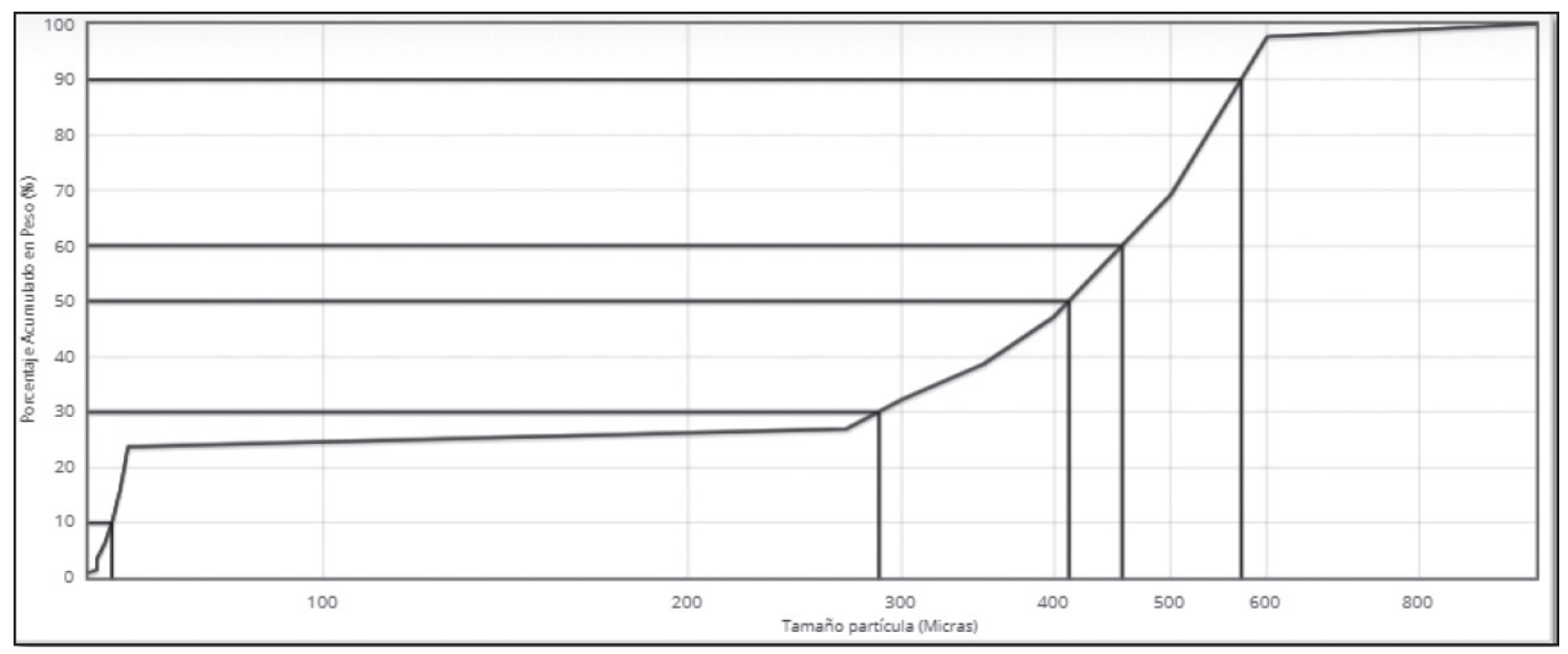

Figura 12. Curva acumulativa de distribución granulométrica de la muestra de interés. (Beltrán \& Carvajal, 2018).

Tabla 9. Variables granulométricas para la selección y diseño de mecanismos de control de arena. (Beltrán \& Carvajal, 2018).

\begin{tabular}{|c|c|}
\hline \multicolumn{2}{|c|}{ Variables granulométricas calculadas para la selección y diseño de mecanismos de control de arena } \\
\hline Tamaño Grano Promedio (micras) & 407.28 \\
\hline Tipo Grano de la Arena & Arena media \\
\hline Percentil D90 (X10) & 70.69 \\
\hline Percentil D70 (X30) & 171.61 \\
\hline Percentil D50 (X50) & 241.67 \\
\hline Percentil D40 (X60) & 267.86 \\
\hline Percentil D10 (X90) & 454.43 \\
\hline Coeficiente de Uniformidad (U) & 3.789 \\
\hline Uniformidad Arena & Arena no uniforme \\
\hline
\end{tabular}

Tabla 10. Mecanismos de control de arena sugeridos. (Beltrán \& Carvajal, 2018).

\begin{tabular}{|c|c|}
\hline \multicolumn{2}{|c|}{ Mecanismos de control de arena sugeridos } \\
\hline Tecnología Convencional de control de Arena Recomendada 1 & Malla premium \\
\hline Tecnología Convencional de control de Arena Recomendada 2 & Empaque con grava y malla \\
\hline Nueva Tecnología de control de Arena Recomendada 3 & Malla Expandible \\
\hline Nueva Tecnología de control de Arena Recomendada 4 & 0.096 \\
\hline Tamaño de grava sugerido con base en el criterio de Saucier (in) & $6 / 8$ \\
\hline Tamaño de grava US. Mesh & \\
\hline
\end{tabular}

Tabla 11. Recomendaciones para la configuración y diseño del mecanismo de control de arena. (Beltrán \& Carvajal, 2018).

\begin{tabular}{|c|c|c|}
\hline \multicolumn{2}{|c|}{ Recomendaciones para la configuración/diseño del mecanismo de control de arena } \\
\hline Modelo (Postulado) & \multicolumn{2}{|c|}{ Rango Tamaño Ranura (in) } \\
\hline Coberly & 0.018 & 0.056 \\
\hline Coberly Actualizado & 0.036 & 0.054 \\
\hline Penberty & 0.032 & 0.048 \\
\hline Regent Energy (Canada Reference) & 0.014 & 0.033 \\
\hline Rango sugerido para el tamaño de las ranuras & $\mathbf{0 . 0 1 4}$ & $\mathbf{0 . 0 5 4}$ \\
\hline
\end{tabular}

